\title{
El empleo de las TIC en la educación superior, una mirada desde la neurociencia educacional
}

\section{The use of ICT in higher education, a view from educational neuroscience}

\author{
Niurys González Cano ${ }^{1}$ \\ niurygc@infomed.cfg.sld.cu \\ https://orcid.org/0000-0001-5332-0065 \\ Hernán Hernández Monzón² \\ ingles5224@ucm.cfg.sld.cu \\ https://orcid.org/0000-0002-1913-0556 \\ Yasleidy González Cano ${ }^{3}$ \\ Ygonzalezcano84@gmail.com \\ https://orcid.org/0000-0002-7115-2234
}

Recibido: 01/07/2020; Aceptado: 01/09/2020

\begin{abstract}
RESUMEN
Las nuevas tecnologías han impuesto cambios significativos en los sistemas educativos, particularmente en la educación superior, donde las nuevas tendencias incluyen el auge extraordinario de las TIC para promover la integración de conocimientos y saberes, el desarrollo de habilidades y el fortalecimiento de valores, donde la neurociencia educacional puede hacer grandes contribuciones. El presente trabajo se propone abordar el proceso de enseñanza y aprendizaje en la educación superior, influenciado por las TIC, con un enfoque basado en neurociencia educacional. El empleo de estas herramientas fomenta el desarrollo de habilidades y competencias en los estudiantes y promueven la capacidad creativa e innovadora de los docentes. La comprensión de las bases neurofisiológicas sobre las que se construye el conocimiento y cómo éste se produce, la socialización, el entorno, la motivación, y otras cuestiones inherentes al aprendizaje del estudiante, constituyen aportes de la neurociencia educacional al perfeccionamiento del proceso docente en las universidades.
\end{abstract}

Palabras clave: Tecnologías de la Información y la Comunicación (TIC), neurociencias, neurociencia educacional

\footnotetext{
${ }^{1}$ Profesor Asistente. Universidad de Ciencias Médicas de Cienfuegos. Facultad de Ciencias Médicas "Dr. Raúl Dorticós Torrado". Cienfuegos, Cuba.

2 Profesor Asistente. Universidad de Ciencias Médicas de Cienfuegos. Facultad de Ciencias Médicas "Dr. Raúl Dorticós Torrado". Cienfuegos, Cuba.

3 Profesor instructor. Clínica Estomatológica de Especialidades Área II, Cienfuegos, Cuba.
} 


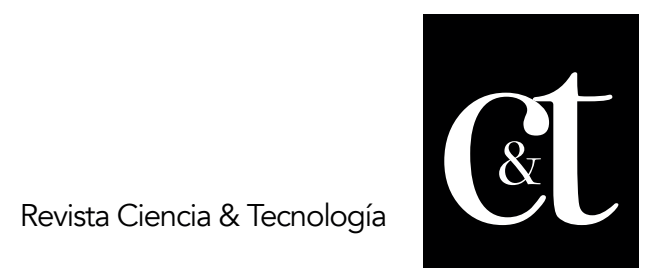

No. 28,31 de octubre de 2020

ISSN impreso: 1390 - 6321

ISSN online: 2661 - 6734

\begin{abstract}
New technologies have imposed significant changes in educational systems, particularly in higher education, where new trends include an extraordinary rise of ICT to promote the integration of knowledge, the development of skills and the strengthening of values, where the educational neuroscience can make great contributions. The present work aims to address the teaching and learning process in higher education, influenced by ICT, with an approach based on educational neuroscience. The use of these tools encourages the development of skills and competencies in students and promotes the creative and innovative capacity of teachers. The understanding of the neurophysiological bases on which knowledge is built and how it is produced, the socialization, the environment, motivation, and other issues inherent to student learning, constitute a contribution of educational neuroscience to the improvement of the teaching process in universities.
\end{abstract}

Keywords: Information and Communication Technologies (ICT), neurosciences, educational neuroscience

\title{
Introducción
}

Las nuevas tecnologías han impactado en los sistemas educativos en todos los niveles de enseñanza, particularmente en la educación superior, donde han impuesto cambios significativos, a tono con las sociedades contemporáneas.

El contexto mundial actual se encuentra marcado por la concentración de las riquezas contra depauperación de las masas, la existencia de un domino económico, militar y cultural del mundo por un pequeño grupo, la crisis del pensamiento social progresista, pérdida de la identidad social y cultural, el sometimiento de la ciencia a la tecnología y de ambas al dinero, globalización, deshumanización, entre otras adversidades, las Tecnologías de la Información y la Comunicación (TIC) han alcanzado un auge extraordinario.

En este escenario, la educación superior ha sufrido trasformaciones. Las tendencias actuales se basan en la expansión cuantitativa con un incremento de la tasa de escolarización superior a expensas de los países desarrollados, la diversificación de estructuras y formas académicas, con una mayor oferta educativa privada, así como la internacionalización, donde sin dudas, el nivel de desarrollo económico de un país constituye una determinante.

En los países desarrollados, los organismos financieros internacionales, basados en un enfoque economicista, conciben la educación superior como parte de los problemas económicos, y lejos de fomentar políticas educacionales públicas, fomentan la privatización. La realidad de los países del tercer mundo es que la universidad sufre una profunda crisis de credibilidad por el pobre apoyo estatal, la falta de voluntad política de los gobiernos, el neoliberalismo y la privatización.

En el mundo de hoy, las tecnologías juegan un rol importante en la formación permanente de los profesionales actuales y futuros que integran la sociedad, mostrando competencias investigativas y habilidades tecnológicas facilitadoras de la construcción de nuevos conocimientos científicos como respuestas a las necesidades 


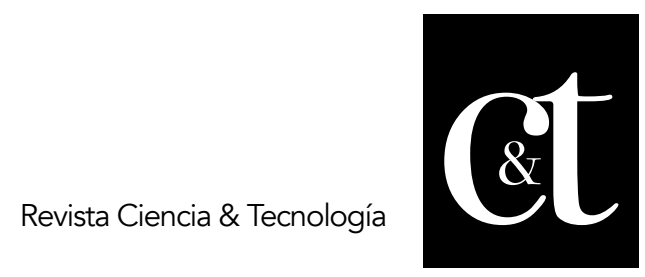

No. 28, 31 de octubre de 2020

ISSN impreso: 1390 - 6321

ISSN online: 2661 - 6734

propias de la sociedad, generadora de una cultura cada vez más exigente, para lo cual el autodesarrollo y la transformación social adquieren un gran significado y sentido social (Cárdenas, Guerra y Soler, 2017).

Hoy día, la formación de profesionales de las diferentes ramas precisa el concurso de las más novedosas ideas y contribuciones sustentadas en la propia ciencia. En este punto, han tenido un auge extraordinario las neurociencias, las que ofrecen una perspectiva renovadora en materia de educación y que ha despertado el interés de muchos investigadores de las ciencias pedagógicas.

Las neurociencias son reconocidas por su gran diversificación, sobre todo en las últimas décadas y con el impulso de los avances tecnológicos. Están integradas por varias disciplinas dedicadas al estudio del funcionamiento de las células nerviosas interconectadas y conformando las vías de señalización nerviosas, lo que ha permitido esclarecer el funcionamiento de la mente. Esta es la base de la percepción, acción, emociones, aprendizaje y hasta la conducta de los seres humanos (Zuluaga Gómez, 2018; Delgado Reyes, 2017).

El origen de las neurociencias se remonta a la década de los ochenta con las contribuciones de Alexander Romanovich Luria (1973, 1980), conocido como padre de la neuropsicología, quien hace importantes aportes en la comprensión del funcionamiento cerebral como producto de la interacción de diversas unidades funcionales, y que le permiten al ser humano desarrollar la actividad mental y comportamental (Lepe Martínez et al, 2017).

Del estrecho vínculo que relaciona neurociencias con aprendizaje se desprende que cuando aprendemos el cerebro cambia su forma, que la experiencia moldea nuestro plástico y flexible cerebro y que el aprendizaje organiza y reorganiza el cerebro (Carminati de Limongelli y Waipan, 2012). En este punto de intersección entre neurociencias y pedagogía encuentra su origen la neurociencia educacional o neuroeducación.

En los sistemas educativos, el empleo racional de las TIC promueve la integración de conocimientos y saberes, el desarrollo de habilidades y el fortalecimiento de valores. El logro de este propósito dependerá de la apropiación de estas herramientas por parte de los educandos en la gestión de su propio aprendizaje, de la misma manera que, los docentes deberán apelar a su capacidad creadora e innovadora para rediseñar el aprendizaje a distancia, utilizando racionalmente los recursos tecnológicos disponibles.

En este sentido, las neurociencias pueden hacer importantes contribuciones partiendo del argumento que cerebro y mente constituyen una unidad indisoluble. El presente trabajo se propone abordar el proceso de enseñanza y aprendizaje en la educación superior, influenciado por la incursión masiva de las TIC, desde una perspectiva renovadora basada en las contribuciones de la neurociencia educacional.

\section{Desarrollo}

La educación del ser humano, a través de la historia ha sido objeto de cambios y transformaciones, de ahí que los representantes del gremio han tenido a su cargo el estudio incesante de nuevos métodos, medios, modelos, técnicas y otros componentes para el aprendizaje: "El protagonismo de los educadores dentro de la 


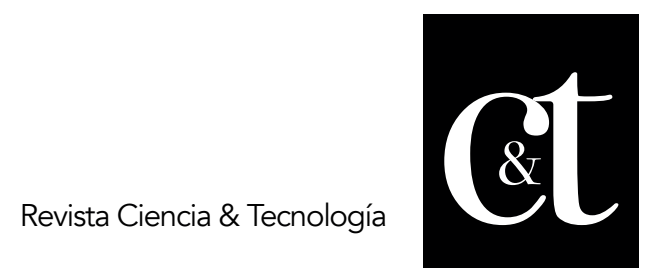

No. 28, 31 de octubre de 2020

ISSN impreso: 1390 - 6321

ISSN online: 2661 - 6734

sociedad, consiste en la búsqueda de mejores estrategias para educar, poniendo en práctica los medios apropiados para las transformaciones." (Campos, 2010).

En la innovación o transformación de la educación y de la práctica pedagógica, lo primero consiste en entender qué será transformado. Puesto que el ser humano está dotado no solamente de habilidades cognitivas, de razón, sino también de habilidades emocionales, sociales, morales, físicas, incluso espirituales, es en el propio individuo donde concuerdan todas ellas, integradas en el cerebro. (Campos, 2010).

En este sentido, han venido desarrollándose las neurociencias, las que han venido diversificándose a través de nexos con otras ciencias como la psicología, la sociología, la educación, la economía, entre otras ramas. Específicamente en el campo de la neurociencia educacional o neuroeducación, se postula que "en el cerebro humano se encuentra la respuesta para la transformación y al mismo tiempo será transformado tanto el cerebro del educando como el del estudiante." (Campos, 2010).

Sobre la base de ese principio, en la Educación Superior, el estudiante constituye el objeto y a la vez sujeto del aprendizaje, por lo que la neurociencia educacional ofrece una perspectiva novedosa en este sentido. Del estrecho vínculo que relaciona las neurociencias con aprendizaje se desprende que cuando se aprende el cerebro cambia, que la experiencia también es importante para moldear el plástico y flexible cerebro, y que el aprendizaje organiza y reorganiza el cerebro (Carminati de Limongelli y Waipan, 2012).

Ha llegado a establecerse la semejanza del funcionamiento del cerebro humano con el de una orquesta. Mediante la interacción de las diferentes áreas del cerebro se integran diversas funciones puesto que existe una íntima conexión entre sus componentes, permitiendo así organizar y seleccionar lo adecuado ante una tarea requerida. Este complejo proceso constituye la base para seleccionar, almacenar y guardar una información nueva, que en la medida que sea recuperada podrá transformarse en aprendizaje (Campos, 2010).

Así, se van desarrollando las habilidades y capacidades, como resultado de un cerebro en constante aprendizaje y que a la vez se desarrolla tanto morfológica como funcionalmente. "Las habilidades y capacidades cognitivas, de la esfera social, emocional, moral y física necesitan ser aprendidas, desarrolladas, practicadas y utilizadas, para conformar y consolidar las bases de todos los conocimientos posteriores." (Campos, 2010).

En la vida de un individuo, es imprescindible la comprensión del aprendizaje, qué es, cómo se produce y cómo se pueden mejorar los procesos, tanto en el orden individual como en lo social. Los avances de la ciencia hoy día, han permitido conocer que durante el desarrollo del sistema nervioso, el cerebro, como órgano central, va cambiando tanto estructural como funcionalmente.

Desde el punto de vista biológico, a lo largo de este proceso, se van estableciendo cambios en las conexiones neuronales como producto del aprendizaje y la interacción con el ambiente que nos rodea. Estas modificaciones se basan fundamentalmente en el aumento del número de sinapsis neuronales y a esta capacidad del cerebro, se le denomina plasticidad cerebral. 


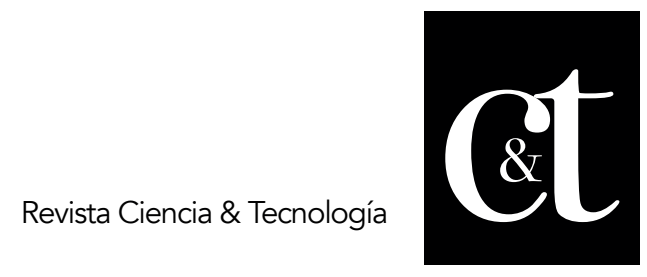

No. 28, 31 de octubre de 2020

ISSN impreso: 1390 - 6321

ISSN online: 2661 - 6734

La plasticidad cerebral, como ha sido expresado por los neurocientíficos, es la cualidad del cerebro humano donde se encuentra la respuesta de como los conocimientos y habilidades adquiridas no son estáticas, sino que están en constante cambio. Se plantea que aprender es bueno para desarrollar el cerebro (Machado, 2016).

En lo que concierne a la propia ciencia cognitiva, cuando se está construyendo un aprendizaje se va estableciendo paulatinamente una red de conexiones neuronales, donde incluso, aquellas neuronas aletargadas contribuyen a consolidar esa red, como redes Hebbianas (Carminati de Limongelli y Waipan, 2012). Estos ensambles que se van estableciendo favorecen el crecimiento, ampliación y potenciación de las redes neuronales, que son la base del aprendizaje.

También la plasticidad cerebral brinda la posibilidad de explicar las infinitas potencialidades del cerebro humano, argumentado desde el diseño de su morfología y función, donde se dan las condiciones propicias para que el medio y sus influencias lo lleven a alcanzar las más altas expresiones (Carminati de Limongelli y Waipan, 2012). En la actualidad las neurociencias aportan cada vez nuevos conceptos a la tarea de "modelar cerebros".

La interacción social también es parte inseparable del proceso de modelación del cerebro humano. Así lo han reflejado varios exponentes de la Psicología basados en la teoría Vygotskiana, que concibe lo social como un factor fundamental y un espacio imprescindible para el aprendizaje a través de la relación que el sujeto entabla con el contexto y con sus semejantes. Así se modela el propio sujeto y se desarrollan las funciones psicológicas superiores.

En un ambiente enriquecido por desafíos y actividades estimulantes se promueve el conocimiento. Tanto es así, que actualmente se habla del nuevo reto para la educación enfocado desde las neurociencias, donde las TIC han provocado una verdadera revolución dentro del conocimiento, donde la figura del profesor, sigue siendo imprescindible, no solo porque ofrece el contacto humano, el ejemplo, el afecto, la inspiración, la mirada social y la motivación, sino porque fomenta los valores en los estudiantes (Machado Trujillo, 2016).

Se ha planteado que la tecnología no reemplaza el componente humano, ni social. Los educadores y filósofos han considerado el influjo del ambiente social tanto en procesos de aprendizaje como también en la configuración del comportamiento del ser humano. Se ha llegado a plantear teorías sobre el aprendizaje social y la influencia del ambiente sociocultural en estos procesos, argumentado sobre bases neurocientíficas (Barrios-Tao, 2016 y Martínez-González et al., 2018).

En los últimos años han ido surgiendo contribuciones y modelos teóricos desde la neuropsicología, que han dado lugar a la aparición de la neuroeducación o neurociencia educacional. Se trata de un campo científico interdisciplinar que estudia la interacción entre los procesos neuronales, psicológicos y la educación con el propósito de mejorar el proceso de enseñanza y aprendizaje en el estudiante (Martínez-González et al., 2018).

Esta rama de las neurociencias, se ha propuesto profundizar en el conocimiento de las múltiples maneras que tiene el cerebro humano para aprender, utilizando varias estrategias y elementos del entorno. Se habla de las múltiples inteligencias que 


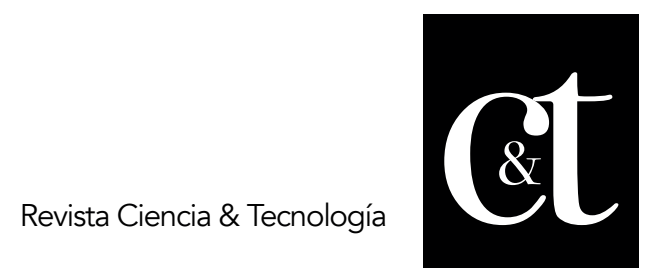

No. 28, 31 de octubre de 2020

ISSN impreso: 1390 - 6321

ISSN online: 2661 - 6734

conforman el cerebro humano, que están interconectadas entre sí pero que a la vez pueden trabajar de manera independiente y tener un nivel individual de desarrollo (Barbón Álvarez, 2016).

Es necesario considerar la filosofía de las Inteligencias Múltiples al esquematizar el trabajo, al proponer diferentes aprendizajes o al programar las actividades que se llevan a cabo en el aula, lo que permitirá que los alumnos utilicen diferentes recursos, provenientes de sus múltiples inteligencias y también desarrollen sus capacidades (Barbón Álvarez, 2016).

Se plantea que el cerebro humano también tiene estilos de aprendizaje, lo que debe ser interpretado por los docentes para planificar y realizar sus actividades explorando algunos de estos estilos de aprendizaje, por ejemplo, el visual, el auditivo, el lingüístico o el lógico. La enorme capacidad de aprender del cerebro humano de estas diversas maneras, proporciona al educador un abanico de ideas y alternativas para proponer un aprendizaje, facilitando el desarrollo de todas las habilidades de pensamiento de los alumnos.

El cerebro de todo ser humano está programado genéticamente para aprender, procesar, consolidar y recordar un aprendizaje, y los sistemas y funciones involucrados en este proceso son los mismos en todos los seres humanos, sería importante que el educador considerara que el alumno también tiene la capacidad de aprender de manera reflexiva, impulsiva, analítica, global, conceptual, perceptiva, motora, emocional, intrapersonal e interpersonal (Campos, 2010). De ahí que se tenga en cuenta la diversidad en el aprendizaje.

El cerebro para aprender necesita percibir y codificar una información, la que se recibe a través de sus recursos multisensoriales, el cuerpo, la motivación y todos los conocimientos previos almacenados en un sistema de memoria en especial. A partir de allí, se desencadena una serie de acontecimientos a nivel neurológico como, por ejemplo, la activación del mecanismo de atención, que permitirá que el alumno procese la información más relevante ignorando otros estímulos (externos o internos) y empiece a adquirir de manera directa o indirecta el aprendizaje (Campos, 2010).

Todos los recursos deben ser confeccionados para que el nuevo aprendizaje sea adquirido y se desarrollen nuevas conexiones sinápticas (y nuevas capacidades). Como el aprendizaje se caracteriza por la habilidad de adquirir nuevas informaciones (Gazzaniga, Ivry, \& Mangun, 2002) es de fundamental importancia que el educador no sólo propicie verdaderas oportunidades de entendimiento de la propuesta de aprendizaje sino también que se certifique que el alumno la está incorporando de manera adecuada.

Para ello, la retroalimentación es un excelente recurso, se debe escuchar a los alumnos, realizar pequeños ejercicios a manera de evaluación, sin mencionarla o hacer otra actividad que permita saber qué entendieron los alumnos. Estas actividades le darán al maestro la idea de cuánto han elaborado el conocimiento y de qué forma lo hicieron (Campos, 2010).

Las discusiones grupales, los debates, la lectura, entre otras, son magníficas actividades para involucrar el aprendizaje explícito tanto como el aprendizaje implícito. Ese último caracterizado por el empleo de metáforas, proyectos, juegos, 


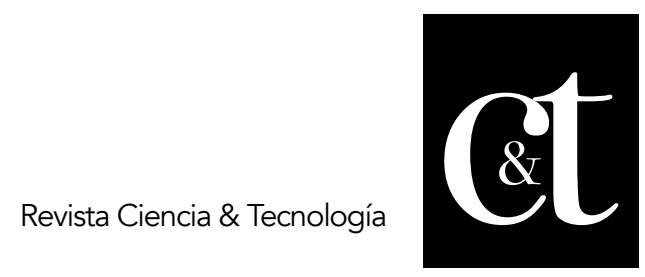

No. 28,31 de octubre de 2020

ISSN impreso: 1390 - 6321

ISSN online: 2661 - 6734

experiencias, dramatizaciones, grabaciones, etc. En esta etapa el maestro debe desempeñar un papel básico de mediador.

El tipo de información que fue retenida, la manera en que fue codificada, archivada y luego evocada va a permitir que el aprendizaje se haga real, significativo y funcional (Campos, 2010). Recordar esta secuencia de acontecimientos a la hora de planificar las actividades docentes, permitirá al educador vincular su práctica pedagógica a la fisiología del aprendizaje del cerebro, contribuyendo significativamente con el promover, desarrollar y fortalecer la red de conexiones neuronales.

Desde la neurociencia educacional, una vez que el maestro entienda las particularidades del sistema nervioso y del cerebro y logre relacionar este conocimiento con el comportamiento de sus alumnos, su propuesta de aprendizaje, su actitud, el ambiente del aula, entre otros factores, puede ser el paso inicial en la formación y capacitación docente que marcará la diferencia en la calidad de la educación (Campos, 2010).

También las neurociencias tienen en cuenta el contexto, que es cambiante y que determina una validez transitoria a los modelos. Los hechos son diferentes en todos los contextos. Por tanto, recontextualizar una idea, implica su reelaboración dentro de marcos de referencia diferentes. "Lo cultural, lo social, lo histórico, los paradigmas conceptuales sobre los cuales se representa la idea son los generadores directos de la crisis de representación, sobre la cual evoluciona y se transforma el universo." (Zuluaga Gómez, 2018).

La realidad cotidiana se caracteriza por un auge extraordinario de herramientas tecnológicas que han abierto inmensas posibilidades, como ejemplo, la internet, las plataformas virtuales incluso, el surgimiento de nuevos dispositivos móviles que han provocado una verdadera revolución en las últimas dos décadas.

Las TIC, han logrado un auge extraordinario como recursos para el aprendizaje. Han demostrado ser herramientas versátiles al facilitar la ubicuidad de las comunicaciones, la inmediatez de los procesos informáticos y la simplicidad de los procesos de creación y difusión de la información. Gracias a ellas, los individuos han adquirido nuevos comportamientos y han surgido nuevas necesidades vinculadas a estas tecnologías (Machado Trujillo, 2016).

Por otro lado, otras actividades han sido transformadas de manera significativa. Por ejemplo, el caso de la mensajería instantánea WhatsApp, también los mensajes de texto por móvil o un correo electrónico, con los que la información es extremadamente rápida y se logra acceder de manera individual o colectiva. Son recursos de gran utilidad en diferentes enseñanzas que deberán ser explotadas por los docentes, a tono con el contexto.

Ante el nuevo y cambiante escenario tecnológico es evidente que los elementos más usados no son solo herramientas, más bien "responden a la recreación que hacen las personas del mundo, convirtiéndose así en agentes de cambio (...), las innovaciones tecnológicas no suceden en abstracto, sino que dependen del contexto, del estado del conocimiento, el entorno institucional, industrial, económico..." (Machado Trujillo, 2016).

\section{El empleo de las TIC en la enseñanza superior}




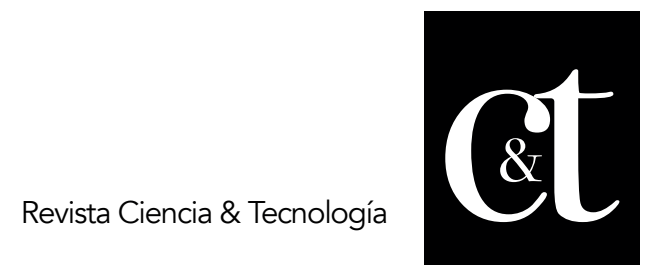

No. 28, 31 de octubre de 2020

ISSN impreso: 1390 - 6321

ISSN online: 2661 - 6734

En la enseñanza universitaria, el proceso de formación de los profesionales, demanda cada vez nuevas estrategias y métodos acordes con las exigencias de los estudiantes, que emanan del propio contexto en el que se desarrollan, impregnado de las tecnologías más novedosas. Ante este complejo escenario, se vincula enseñanza, aprendizaje e instrucción, tal y como demanda la sociedad contemporánea.

La incursión masiva de las TIC como herramientas y medios para potenciar los procesos de enseñanza y aprendizaje es la realidad cotidiana de los sistemas educativos y planes de formación actuales. Acorde con un mundo cada vez más avanzado en cuestiones tecnológicas, y con una racionalidad cada vez más tecnócrata, la esfera educativa apuesta firmemente por incluir estas tecnologías que abren un abanico de nuevas posibilidades para enriquecer las metodologías docentes y facilitar el aprendizaje del alumnado (Machado Trujillo, 2016).

En los últimos tiempos "las TIC han irrumpido con fuerza en las aulas. Son evidentes las múltiples iniciativas y estudios que muestran una amplia gama de proyectos sobre su uso como medios y herramientas educativas." (Machado Trujillo, 2016) Otros autores promueven su empleo como competencia transversal para el desarrollo de otras, siendo un recurso motivante que sirve para potenciar y estimular el proceso de aprendizaje (Martínez-González et al., 2018).

Sin embargo, también se ha alertado sobre el aprendizaje a través de las TIC que puede tener efectos nocivos en comparación con el empleo de las tecnologías tradicionales. Con frecuencia los ambientes tecnológicos sobrecargan los recursos cognitivos del alumnado y lejos de potenciar y facilitar los aprendizajes, los dificultan. El uso de estas tecnologías como herramientas educativas, debe por tanto ser dosificado, para que generen una carga cognitiva que no interfiera sobre los recursos cognitivos del alumnado (Machado Trujillo, 2016).

El adecuado control de los elementos a interactuar, la forma en la que se presenta la información y evitar sobrecargar los materiales con elementos sin relevancia, son claves para no sobrecargar la memoria de trabajo y facilitar la comprensión y adquisición de conocimientos (Machado Trujillo, 2016). Resulta indispensable la adecuada orientación del profesor hacia una búsqueda concreta de la información en los entornos virtuales.

Se hace referencia a la lectura hipertextual que con frecuencia se muestra en entornos web, con lo que se accede a mayor cantidad de información. En ella la atención se verá más sobrecargada de estímulos y de enlaces, la jerarquía de contenidos puede ser bastante difusa y enrevesada, y en definitiva puede desviarse la información buscada en inicio (Machado Trujillo, 2016).

Al realizar una comparación entre la lectura hipertextual y el texto físico, los procesos cognitivos se desarrollan de forma distinta y la memoria de trabajo se verá más sobrecargada en el caso de la lectura en un entorno virtual online. Este elemento debe ser valorado con precisión por los docentes en el diseño de cada actividad docente, donde las TIC sean el recurso del aprendizaje disponible.

De manera general el procesamiento cognitivo del alumnado es bien distinto cuando trabaja la información y resuelve tareas en entornos físicos frente a entornos virtuales, cuestión que no se puede pasar por alto en el diseño de situaciones de 


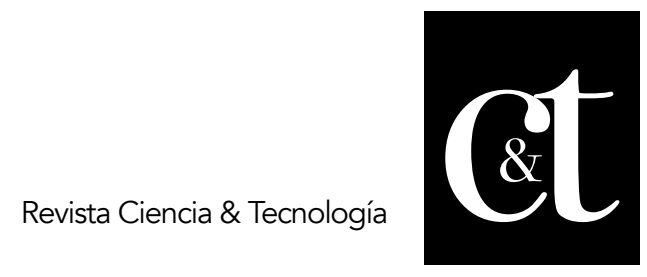

No. 28,31 de octubre de 2020

ISSN impreso: 1390 - 6321

ISSN online: 2661 - 6734

aprendizaje. "Está claro que las TIC están desarrollando una mayor capacidad multitarea en las personas, pero tratan de forma muy diferente la atención, la memoria de trabajo y la comprensión que otros métodos tradicionales", y se debe trabajar tomando conciencia de ello (Machado Trujillo, 2016).

Así, los sistemas educativos deberán reorganizarse y hacer una selección adecuada de las TIC para responder a las demandas de un escenario social cada día más tecnológico. El uso racional de estas, es tarea de quienes diseñan, ejecutan y controlan el proceso de enseñanza y aprendizaje, teniendo en cuenta, las particularidades de los estudiantes y sus estilos para apropiarse del conocimiento, es decir, la atención a diferencias individuales.

En la educación superior, la neurociencia educacional, puede hacer grandes aportes si se logra identificar al aprendizaje como una expresión que tiene lugar en múltiples niveles al mismo tiempo, y se pueden identificar como eventos de aprendizaje producidos desde un estrato biológico con procesos neurofisiológicos, por medio de un estrato cognitivo que atiende al pensamiento racional y de conocimiento, así como un estrato sociocultural donde las herramientas junto con sus prácticas producen experiencias de aprendizaje (Reimann, 2016).

Este conocimiento contribuye al perfeccionamiento de los docentes de las universidades, quienes tienen a su cargo un importante reto en las sociedades modernas, marcadas por la globalización, el sometimiento de la ciencia a la tecnología, y de estas al dinero, la individualización, los cambios ambientales, entre otras tendencias, incluidas las TIC.

"La tecnología impacta en el aprendizaje transformando los medios a través de los cuales se accede a la educación." (Corona Ferreira et al., 2019). Estas herramientas hoy día han alcanzado un auge extraordinario pues el empleo de nuevos recursos tecnológicos, ha permitido diseñar el acercamiento a los alumnos para orientar, desarrollar y dar seguimiento al aprendizaje, incluso fuera del aula tradicional.

Los docentes tienen la tarea no solo de orientar adecuadamente los contenidos de las materias y dosificar las tareas docentes, sino también el planteamiento de tareas investigativas que motiven al alumnado, siempre que la información esté al alcance de estos, en aquellos sitios donde puedan acceder sin dificultad. Cuando el acceso a la información en determinadas páginas web o plataformas interactivas se torna difícil, deja de ser un incentivo para los estudiantes.

De esta manera, la neurociencia educativa concibe la necesidad del estímulo y la motivación para lograr un mejor aprendizaje, lo que favorece la capacidad de incorporar los nuevos conocimientos en la construcción de un pensamiento desarrollador, siempre teniendo en cuenta las potencialidades del cerebro humano (Falconí et al., 2017). Desde esta óptica se puede llegar a entender el origen del deseo por aprender, en la medida en que se logren determinar las causas que motivan al alumno darle la significancia al aprendizaje.

De manera que la orientación de actividades motivadoras, dosificadas y concretas, contribuirán a una adecuada autopreparación del estudiante. La independencia cognoscitiva del estudiante no puede ser lograda sin el estímulo constante, lo que sin lugar a dudas constituye un verdadero reto para los docentes. 


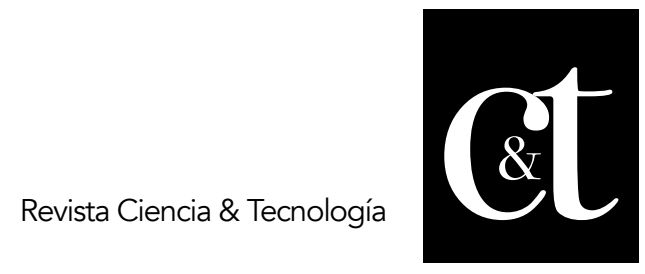

No. 28, 31 de octubre de 2020

ISSN impreso: 1390 - 6321

ISSN online: 2661 - 6734

Otro elemento de interés para la neurociencia educacional es la socialización del conocimiento, no solo entre el estudiante y el profesor sino también mediante el intercambio grupal. En este sentido las TIC ofrecen un amplio espectro de oportunidades, sobre todo para la enseñanza a distancia y en entornos virtuales. Un ejemplo concreto, los grupos WhatsApp, que evidencian una forma de socializar el conocimiento entre los estudiantes y entre estos y el profesor.

Autores como Torrijos López, en su trabajo: "La clase invertida como garantía de éxito en las clases online", resalta la tendencia actual de crecimiento en lo relacionado con la educación online, que ofrece numerosos beneficios a docentes y alumnos. La autora propone que las clases online deben tener como objetivo la metodología de la clase invertida, conocido como flipped lessons (Torrijos López, 2019).

Según este método las partes teóricas pueden ser grabadas sin presencia de los alumnos, mientras que las clases en las que estos se conectan se trabaja con la teoría que ha debido ser visualizada previamente por éstos, así en el resultado sería una interacción total entre alumnos y profesores en el tiempo concebido, aprovechándose el máximo rendimiento. Ofrece la ventaja del profesor poder escuchar a los estudiantes y sus explicaciones (Torrijos López, 2019).

La experiencia de otros autores como Yáñez Martínez ha demostrado que presencia digital constituye un reto para mantener la motivación y cercanía entre alumnos y docentes de enseñanzas online, la que ha ido desarrollando nuevas habilidades. Estas estrategias giran en torno a hacer sentir más cercanos los docentes, a pesar de la distancia; conocer y transmitir familiaridad creando un ambiente horizontal de aprendizaje grupal y autoevaluación (Yáñez Martínez, 2019).

De esa forma, las actividades están pensadas para la reflexión en la identidad personal y visual. Pueden ser valoradas las distintas posibles soluciones a un mismo planteamiento, en algunos casos la muestra en grupo se hace imprescindible para la finalización de la actividad. Esto ayuda a generar cercanía entre ellos y fomenta el deseo de superación (Yáñez Martínez 2019).

Es importante destacar la figura del profesor en el proceso de socialización, no solo porque orienta y guía hacia el conocimiento, sino porque refuerza el componente educativo del proceso y el fortalecimiento de valores. Para el docente de la educación superior, el actual contexto marcado por la tecnología, representa un verdadero reto que deberá enfrentar en su rol de mediador en el proceso de enseñanza y aprendizaje.

El escenario tecnológico, pone a prueba la capacidad creativa e innovadora del profesor, así como el desarrollo de competencias en el orden tecnológico, curricular e investigativo, en función de los estudiantes. En este sentido, la neuroeducación es la disciplina de las neurociencias que facilita la formación a los profesores para que sepan transferir la información neurocientífica al ámbito educativo.

También en los estudiantes se potencializan las competencias básicas en ciencia y tecnología, competencias digitales, competencias de aprender a aprender, competencias sociales y cívicas, entre otras (Martínez-González et al., 2018). Todas de extraordinaria importancia durante el proceso de formación como futuros 


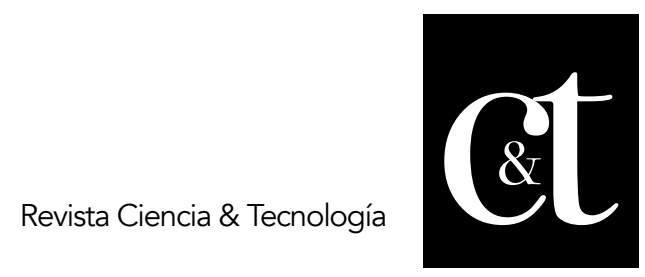

No. 28,31 de octubre de 2020

ISSN impreso: 1390 - 6321

ISSN online: 2661 - 6734

profesionales. Del desarrollo de estas competencias dependerá la calidad del profesional egresado de las universidades.

En materia de neurociencia educacional se concibe la creatividad como un elemento esencial en el proceso de enseñanza y aprendizaje. La creatividad, ha sido definida como "la capacidad que poseen las personas para producir ideas originales y desde éstas producir materiales nuevos, teniendo en cuenta el contexto social en el que ocurren dichas innovaciones" (Rodríguez-Muñoz, 2011).

Asumiendo las múltiples concepciones acerca del proceso creativo, sí existe consenso que se inicia con la necesidad de interpretar una situación que supone la satisfacción de una necesidad, un fallo o hueco vacío en el funcionamiento de algo, la solución de un problema nuevo que no ha surgido antes y cuyos heurísticos no son válidos, y el descubrimiento de un problema. Una primera fase del proceso creativo implicaría cierta sensibilidad a los problemas, actitud crítica, búsqueda de información y de novedad (López Fernández y Llamas Salguero, 2018).

\section{Conclusiones}

El impacto de las nuevas tecnologías en los sistemas educativos, sin dudas promueve la búsqueda de métodos y alternativas para potenciar el aprendizaje. En la enseñanza superior, el empleo de las TIC ha provocado una verdadera revolución dentro del conocimiento, convirtiéndose en una valiosa herramienta para el desarrollo de habilidades y competencias en los estudiantes, a la vez que promueven la capacidad creativa e innovadora de los docentes.

La comprensión de las bases neurofisiológicas sobre las que se construye el conocimiento, la forma en que este se produce, la socialización, el papel del entorno, la importancia de la motivación, entre otras cuestiones inherentes al aprendizaje del estudiante, constituyen aportes de la neurociencia educacional para el perfeccionamiento del proceso docente en las universidades.

\section{Referencias bibliográficas}

Barbón Álvarez, M. (2016). El desarrollo de la creatividad y las inteligencias múltiples en niños de educación infantil mediante el uso de metodologías tradicionales e innovadoras en el aula. Tesis de master. Universidad Internacional de la Rioja.

Barrios-Tao, H. (2016). Neurociencias, educación y entorno sociocultural. Educación y Educadores, 19(3), 395-415. doi: 10.5294/ edu.2016.19.3.5

Campos, A.L. (2010). Neuroeducación: uniendo las neurociencias y la educación en la búsqueda del desarrollo humano. Revista digital La educación, No.143.

Cárdenas Zea, M. P., Guerra González, C. y Soler Pellicer, Y. (2017) Impacto Social de la formación de competencias investigativas mediada por la tecnología en profesionales de la universidad técnica estatal de Quevedo. Revista Didáctica y educación, 8(2)

Carminati de Limongelli, M. y Waipan, L., (2012). Integrando la neuroeducación al aula. Editorial Bonum. 1a ed. 160.

Corona Ferreira, A., Altamirano, M., López Ortega, M.A. y González González, O. A., (2019). Analítica del aprendizaje y las neurociencias educativas: nuevos retos en la integración tecnológica. Revista Iberoamericana de Educación, 80(1), 31-54 


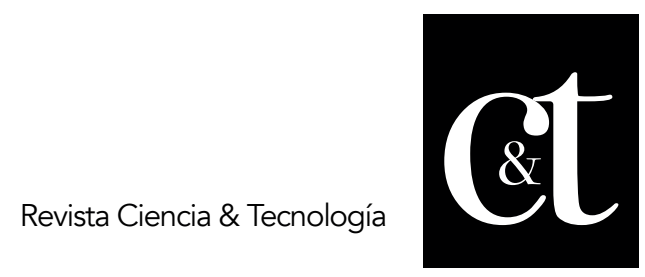

No. 28, 31 de octubre de 2020

ISSN impreso: 1390 - 6321

ISSN online: 2661 - 6734

Delgado Reyes, A.C. (2017). Neurociencia y Psicología. Tempus Psicológico1 (1),127144. doi: https: //doi.org/10.30554/tempuspsi.1.1.2150.2018

Falconí, A., Alajo, A., Cueva, M., Mendoza, R. Ramírez, S. y Palma, E. (2017). Las neurociencias. Una visión de su aplicación en la educación. Revista Órbita Pedagógica 4(1), 61-74

Gazzaniga, M., Ivry, R., \& Mangun, G. (2002). Cognitive Neuroscience: The Biology of the Mind. Estados Unidos.

Lepe Martínez, N.F., Ramos-Galarza, C., Ramos, V., Jadán-Guerrero, J., ParedesNúñez, L., Gómez-García, A. y Bolaños-Pasque, M., (2017). Conceptos fundamentales en la teoría neuropsicológica. Revista Ecuatoriana de Neurología, 26(1), 53-60

López Fernández, V. y Llamas Salguero, F. (2017). Neuropsicología del proceso creativo. Un enfoque educativo. Revista Complutense de Educación, 29 (1) 113127.

Machado Trujillo, C. (2016). Aprendizaje con nuevas tecnologías: una mirada desde la Neurociencia y la Psicología Cognitiva. III Jornadas Iberoamericanas de Innovación Educativa en el ámbito de las TIC. Las Palmas de Gran Canaria 17-18 de noviembre de 2016

Martínez-González, A. E., Piqueras, J. A., Delgado, B. y García-Fernández, L. M. (2018). Neuroeducación: aportaciones de la neurociencia a las competencias curriculares. Publicaciones, 48(2), 23-34. doi: $10.30827 /$ publicaciones.v48i2.8331

Reimann, P. (2016). Connecting learning analytics with learning research: the role of design-based research. Learning: Research and Practice, 2:2, 130-142. https://doi.org/10.1080/ 23735082.2016.1210198

Rodríguez-Muñoz, F.J. (2011). Contribuciones de la neurociencia al entendimiento de la creatividad humana. Arte, Individuo y Sociedad, 23 (2), 45-54.

Torrijos López, E. (2019). La clase invertida como garantía de éxito en las clases online. I Jornada Smart Campus. Universidad Antonio de Nebrija.

Yáñez Martínez, B. (2019). La presencia digital. Creando identidad visual para el aprendizaje y la cercanía. I Jornada Smart Campus, mayo 2019. Universidad Antonio de Nebrija.

Zuluaga Gómez, J.A. (2018). Diálogo en torno a las neurociencias en educación. Neurociencias y educación infantil, Vol. 7 Núm. 1, páginas 86-87. 